\title{
Identification and Field Evaluation of Grape Shoot Volatiles Attractive to Female Grape Berry Moth (Paralobesia viteana)
}

\author{
Dong H. Cha • Satoshi Nojima • Stephen P. Hesler • \\ Aijun Zhang • Charles E. Linn Jr. • Wendell L. Roelofs • \\ Gregory M. Loeb
}

Received: 25 March 2008 /Revised: 21 May 2008 / Accepted: 10 June 2008 / Published online: 23 July 2008

(C) Springer Science + Business Media, LLC 2008

\begin{abstract}
Solid-phase microextraction (SPME) and gas chromatography coupled with electroantennographic detection (GC-EAD) were used to identify volatile compounds from shoots of riverbank grape (Vitis riparia) that attract the female grape berry moth (GBM, Paralobesia viteana). Consistent EAD activity was obtained for 11 chemicals: (Z)-3-hexen-1-yl acetate, (E)-linalool oxide, (Z)-linalool oxide, nonanal, linalool, $(E)$-4,8-dimethyl-1,3,7-nonatriene, methyl salicylate, decanal, $\beta$-caryophyllene, germacrene-D, and $\alpha$-farnesene. In flight-tunnel tests that involved female GBM and rubber septa loaded with subsets of these 11 compounds, we found that both the 11-component blend and a seven-component blend, composed of $(E)$-linalool oxide, (Z)-linalool oxide, nonanal, (E)-4,8-dimethyl-1,3,7-nonatriene, decanal, $\beta$-caryophyllene and germacrene-D, elicited equivalent levels of upwind flight as freshly cut grape shoots. The removal of any of the seven compounds from the seven-component blend resulted in a significant decrease in female upwind flight responses. In a field trial with these two synthetic blends, traps equipped with either blend
\end{abstract}

D. H. Cha $\cdot$ S. Nojima $\cdot$ S. P. Hesler • C. E. Linn Jr.

W. L. Roelofs $\cdot$ G. M. Loeb $(\bowtie)$

Department of Entomology,

NYS Agricultural Experimental Station, Cornell University, Geneva, NY 14456, USA

e-mail: gme1@cornell.edu

\section{A. Zhang}

USDA-ARS-PSI,

Invasive Insect Biocontrol and Behavior Laboratory,

Beltsville, MD 20705, USA

\section{Present address:}

S. Nojima

Department of Entomology, North Carolina State University,

Raleigh, NC 27695, USA captured more female GBM compared to traps baited with hexane only (control), although the number of females caught was generally low. There were no differences in the number of males captured among treatments. Although in flight-tunnel trials, moths readily flew upwind to both grape shoots and rubber septa loaded with the best lures, they landed on shoots but not on rubber septa. Coupled with relatively low field catches, this suggests that additional host finding cues need to be identified to improve trap efficacy.

Keywords Flight tunnel · Field test - Synthetic blend . Paralobesia viteana $\cdot$ Vitis spp. $\cdot$ Host volatiles $\cdot$ Tortricidae

\section{Introduction}

Paralobesia viteana (grape berry moth, GBM) is a tortricid moth native to the eastern United States that specializes on Vitis spp. The larvae of GBM damage grape berries throughout the growing season, which, in addition to reducing yields, predisposes the damaged clusters to pathogens, making this species one of the most important pests of grapes grown in the eastern USA (Slingerland 1904; Goodwin 1916; Dozier and Butler 1929; Gleissner 1943; Taschenberg 1945). On average, two to four generations of GBM occur during a growing season, with the first generation starting with the flight of adults from overwintered pupae around bloom (Taschenberg 1945). The female-produced sex pheromone of this species was identified by Roelofs et al. (1971). Traps baited with the synthetic pheromone are reasonably effective in identifying the start of the first flight of the adults. However, they often fail to predict oviposition on grape clusters by subsequent generations, making the timing of management decisions difficult (Hoffman 1990; Weigle et al. 1999). 
Over the last two decades, an increasing number of studies has reported on the importance of host-plant volatile chemicals in mediating host recognition by herbivores (Visser 1986; Bernays and Chapman 1994; Schoonhoven et al. 1998; Bruce et al. 2005). This volatile-mediated response has been suggested to be more pronounced in specialist than generalist herbivores (Bernays and Chapman 1994), and can occur to very low concentrations of chemicals (Angioy et al. 2003). Several studies have investigated optimal combinations of host volatiles to improve monitoring or trapping of insect pests (e.g., Zhang et al. 1999; Hammack 2003; Blackmer et al. 2004; Leskey et al. 2005; Pinero et al. 2006). Within tortricid moth pests, research on the use of host volatiles has focused on species with moderate to broad diet breadths such as codling moth, Oriental fruit moth, and European grape berry moth. In these cases, a number of relatively common volatile compounds show behavioral activity in the laboratory (Ansebo et al. 2004; Coracini et al. 2004; Hern and Dorn 2004; Natale et al. 2004; Tasin et al. 2005, 2006a, b, 2007). However, lures based on host-plant volatiles have not been successful in the field (e.g., Ansebo et al. 2004; Coracini et al. 2004; Mitchell et al. 2008). One possibility is that the role of odors in location of hosts may be a stronger selective force for specialists than for generalists (Bernays and Chapman 1994). Therefore, the development of a lure based on host-plant volatiles to improve monitoring and management decisions for insect pests may be a more suitable strategy for specialist herbivores such as GBM.

Here, we present results of chemical, electrophysiological, and behavioral experiments that used solid phase microextraction (SPME), gas chromatography-electroantennogram detection (GC-EAD), and flight-tunnel assays, to identify the key volatile chemicals from grape shoots used by female GBM to locate a host. In our previous behavioral study, grape shoots were the most attractive grape tissue to female GBM (Cha et al. 2008). Our goal was to develop a host-plant-based synthetic lure that is as attractive as live grape shoots to female GBM. We also present data from a field trial in which we tested the efficacy of traps baited with synthetic lures.

\section{Methods and Materials}

Insects GBM were reared in cages placed in walk-in environmental chambers at $26^{\circ} \mathrm{C}$ and $60 \% \mathrm{RH}$ under a 18:6 (L:D) photoperiod. Adult moths were fed with $50 \%$ honey and water soaked on cotton. Adults mated freely in rearing cages $(45 \mathrm{~cm} \mathrm{H} \times 77 \mathrm{~cm} \mathrm{~W} \times 45 \mathrm{~cm} \mathrm{D})$ and oviposited on seedless grape (Vitis vinifera, red flame variety). First and second instars were transferred to a diet cup (30 ml, WinCup Inc.) and reared on semi-synthetic diet
(Nagarkatti et al. 2000) that consisted of grapes, pinto beans, and commercially available tobacco hornworm diet (Bio-Serve). To minimize any potential effect of laboratory rearing on the behavior of moths, colonies were reestablished once a year with larvae and pupae collected from commercial vineyards in July or August.

Plants We used a native host species of GBM in northeastern USA, Vitis riparia, to identify candidate volatile host compounds for the synthetic lure. Cuttings were made from $V$. riparia growing in a research vineyard at the Cornell University, New York State Agricultural Experiment Station in Geneva, NY, USA in December 2005. Cuttings were dipped in rooting hormone (Green Light Co., San Antonio, TX, USA) and placed in a planting box filled with moistened Perlite ${ }^{\circledR}$ in a cold room $\left(4^{\circ} \mathrm{C}\right)$, equipped with a heating pad, to facilitate root growth while retarding development of shoots. Once roots were developed, all plants were transplanted to 1-gal pots filled with a mixture of sand, peat moss, and Vermiculite ${ }^{\circledR}$ and moved to a greenhouse with temperatures maintained between $21-26^{\circ} \mathrm{C}$. Supplemental light was provided to extend the day length to $16 \mathrm{~h}$ and pots were fertilized weekly with water-soluble fertilizer (Peters 20-20-20, Scotts-Sierra Horticultural Products Co.). For sampling of volatile chemicals and flighttunnel assays (see below), we used plants that were 2-4 weeks post-bloom, without any flowers or berries, since grape shoots were most attractive to female GBM in our previous flight-tunnel study (Cha et al. 2008).

Adsorbent Sampling We used a push-pull collection system to collect headspace volatiles of live grape shoots. The system was a custom-made, bell-shaped glass chamber (18 cm ID, $10 \mathrm{~L}$ ) with two air-in adapters (7 mm ID) on the top and four air-out adapters (7 mm ID) equally distributed at the bottom wall of the chamber. To accommodate a whole, live potted plant, the glass chamber was placed on two pieces of Pyrex glass with a hole $(2 \mathrm{~cm})$ in the middle so that the vegetative portion of the plant could be sampled. After a plant was set up in the chamber, the chamber was flushed with filtered air $\left(3 \mathrm{~L} \mathrm{~min}^{-1}\right)$ for an hour to replace air inside the chamber with filtered air and to stabilize volatile emission from the plant, because we noticed that handling of the plant during set up temporarily induced release of green leaf volatiles. During the collection, flow meters were used to insure that more filtered air was pushed into the chamber than pulled out through the charcoal filters so as to eliminate possible contamination from outside air. Filtered clean air from a wall-mounted air filtering system (ARS Inc., Gainesville, FL, USA) was pushed into the chamber at 2.5 $\mathrm{L} \mathrm{min} \mathrm{mi}^{-1}$ and volatiles from the headspace of grape shoots were drawn by a vacuum pump onto four activated charcoal filters (ORBO32-small, Supelco Inc., Bellefonte, 
PA, USA) at $0.5 \mathrm{~L} / \mathrm{min} /$ filter. Adsorbent samplings were made from four plants, with collections for each grape plant made over 4 days at room temperature with supplemental light (18:6 L:D). The chamber was washed with acetone, and new ORBO filters were used for a new plant. The volatiles were eluted with $1 \mathrm{ml}$ hexane every $24 \mathrm{~h}$ and then combined. The combined extract was concentrated to $1 \mathrm{ml}$ under a gentle stream of nitrogen gas and kept in a freezer $\left(-20^{\circ} \mathrm{C}\right)$ and subjected to GC-EAD and gas chromatography-mass spectrometry (GC-MS) analyses, and flighttunnel bioassay.

SPME Sampling We used the same glass chamber that was used for adsorbent sampling to collect headspace volatiles of live grape shoots using SPME. Filtered air was pushed into the chamber at $1 \mathrm{~L} \mathrm{~min}^{-1}$. Three of the four air outlets were blocked with rubber septa, while one was $70 \%$ blocked with Teflon tape allowing the SPME fiber to be inserted into a thin stream of headspace volatiles for increased collection efficiency. After a plant was set up in the chamber, we flushed the chamber with filtered air as described above. We conditioned a carboxen/poly-dimethyl siloxane-coated SPME fiber (film thickness $85 \mu \mathrm{m}$; Supelco Inc., Bellefonte, PA, USA) in the GC injector $\left(280^{\circ} \mathrm{C}\right)$ for $5 \mathrm{~min}$ and then inserted the fiber through a small hole on the air-outlet. The fiber was exposed for up to $24 \mathrm{~h}$ to absorb the volatiles, and immediately subjected to GC-EAD or GC-MS analyses. The chamber was washed with acetone before sampling a new plant.

Coupled GC-EAD Analysis Coupled GC-EAD analyses were performed following procedures described previously (Zhang et al. 1999; Nojima et al. 2003). A Hewlett-Packard 5890 Series II gas chromatograph, equipped with a nonpolar EC-1 capillary column $(30 \mathrm{~m} \times 0.25 \mathrm{~mm}$ ID, $0.25 \mu \mathrm{m}$ film thickness; Alltech Associates, Inc., Deerfield, IL, USA) or a polar EC-Wax Econo-Cap capillary column $(30 \mathrm{~m} \times 0.25 \mathrm{~mm}$ ID, $0.25 \mu \mathrm{m}$ film thickness; Alltech) was used for GC-EAD analyses. The oven temperature was programmed from $40^{\circ} \mathrm{C}$ for $5 \mathrm{~min}$ then increased by $15^{\circ} \mathrm{C} \mathrm{min}^{-1}$ to $250^{\circ} \mathrm{C}$. Injector and detector temperatures were set at $280^{\circ} \mathrm{C}$ and $270^{\circ} \mathrm{C}$, respectively. A $0.75-\mathrm{mm}$ ID glass inlet liner (Supelco) was used for SPME sample injection and a 4-mm ID liner (Supelco) for liquid samples. Splitless injection was used with nitrogen as the carrier gas at a flow of $2 \mathrm{ml} \mathrm{min}{ }^{-1}$. The column effluent was split in a ratio of 1:1 in the oven to the flame ionization detector and to the heated $\left(270^{\circ} \mathrm{C}\right) \mathrm{EAD}$ port.

A whole head was removed from a 3-day-old virgin female GBM and both antennae were positioned between two gold wire electrodes that were immersed in two saline-filled micropipettes in a small acrylic holder. We used an EphrussiBeadle insect Ringer as saline (Ephrussi and Beadle 1936).
The moth's head was placed into the tip of one micropipette, and the tips of both antennae, which were wetted with saline containing surfactant $(0.02 \%$ Triton $\mathrm{X}-100)$ for easy insertion, were maneuvered to make contact with the saline in the other micropipette tip. The antennal holder was placed inside a humidified cooling condenser maintained at $10^{\circ} \mathrm{C}$. To compare relative magnitudes of antennal responses, the millivolt values for EAD-active compounds were calculated from GC-EAD recordings performed with SPME and adsorbent sample runs. A minimum of five different antennal pairs (two to three runs/pair) were used to analyze volatiles from shoots of the same four $V$. riparia plants that were subjected to adsorbent sampling.

Chemical Analysis GC-MS was carried out with a Shimadzu GCMS-QP5050A quadrupole mass spectrometer running in the EI (at $70 \mathrm{eV}$ ) scan mode coupled with a Shimadzu GC-17A equipped with a nonpolar DB-1 capillary column $(30 \mathrm{~m} \times 0.25 \mathrm{~mm}$ ID, $0.25 \mu \mathrm{m}$ film thickness; J\&W Scientific, Folsom, CA, USA) or a polar EC-Wax Econo-Cap capillary column $(30 \mathrm{~m} \times 0.25 \mathrm{~mm}$ ID, $0.25 \mu \mathrm{m}$ film thickness; Alltech Assoc.). Helium was used as the carrier gas at a constant flow of $1.0 \mathrm{ml} \mathrm{min}^{-1}$. GC conditions and temperature program were as for the GCEAD analyses. Volatile compounds were identified by mass spectral matches to library spectra as well as by retention time matches to available authentic standards. The EADactive compounds were verified by GC-EAD analysis with authentic standards. Quantification of the relative ratio of the EAD-active compounds was made from the adsorbent collection based on ion abundances from GC-MS analyses.

Chemicals (Z)-3-hexen-1-yl acetate, linalool oxide (1:1 mixture of $(E)$ - and $(Z)$-isomers), nonanal, linalool, methyl salicylate, decanal, $\beta$-caryophyllene, and $\alpha$-farnesene, were obtained from Sigma-Aldrich Inc. (St. Louis, MO, USA), Alfa Aesar (Ward Hill, MA, USA), Fluka (Buchs, Switzerland) or TCI America (Portland, OR, USA). All, except $\alpha$ farnesene (a mixture of various isomers) were greater than 97\% purity. The 4,8-dimethyl-1,3(E),7-nonatriene was synthesized by oxidation of (E)-citral (Zhang et al. 2002) and then by Wittig reaction (Greenwald et al. 1963), and was a mixture of $95 \%(E)$-isomer and $5 \%(Z)$-isomer. Germacrene-D was isolated from golden rod as $91 \%$ germacrene-D and 9\% $\beta$-caryophyllene (by USDA Chemistry Research Unit, Gainesville, FL, USA).

For all flight-tunnel bioassays and field tests, mixtures were prepared in ratios that corresponded to the ratios of compounds found in the collections of $V$. riparia shoots. For flight-tunnel bioassays, the blends with all identified compounds were mixed according to the ratios indicated by live plant samplings and diluted with hexane to $0.108 \mu \mathrm{g}$ of total compounds in $1 \mu \mathrm{l}$ of hexane. Preliminary flight- 
tunnel experiments showed adequate moth responses to this concentration. All subsets of this blend were mixed at the same concentration as the blend with all identified compounds. For the field trial, the blend of compounds tested was diluted to $0.875 \mu \mathrm{g} \mu \mathrm{l}^{-1}$. All the blends were stored in a freezer $\left(-20^{\circ} \mathrm{C}\right)$ between tests.

Flight-Tunnel Assays Five-day-old females from a mating cage were used for flight-tunnel bioassays. Each cohort was set up using 10-15 female pupae (near eclosion) in a Plexiglas mating cage $(30 \mathrm{~cm} \mathrm{H} \times 30 \mathrm{~cm} \mathrm{~W} \times 30 \mathrm{~cm} \mathrm{D})$ and provided with $50 \%$ honey and water. Fifteen to 20 males (one antenna clipped for distinction from females) were added to the cage with a grape cluster. On average, $70 \%$ of females were mated by the fifth day, with copulation peaking on the second day (G. Loeb, unpublished data). The responses of female moths from the mating cage to grape shoots and volatile blends were assessed in a flight tunnel as described in Cha et al. (2008). After each flight-tunnel trial, the male and female moths were returned to the colony to maintain a consistent population level. We did not confirm mating status of females in the flight-tunnel experiments. The flight tunnel was $2 \mathrm{~m}$ in length by $0.6 \mathrm{~m}$ in width and $0.6 \mathrm{~m}$ in height, with a fan installed at the upwind end to create a steady airflow into the tunnel and an exhaust hood at the downwind end to evacuate odor from the flight tunnel. Wind speed was set at $0.25 \mathrm{~m} \mathrm{~s}^{-1}$ at the point of release of moths. A pattern made of dark green paper circles $(10 \mathrm{~cm}$ diameter) was randomly presented both on a white background glass floor and on the glass ceiling below the light source. The upwind and downwind ends of the tunnel consisted of two layers of cheesecloth to prevent escape of moths. Light was provided from above by eight $25-\mathrm{W}$ incandescent bulbs. Light intensity in the tunnel was $25 \mathrm{~lx}$ so as to mimic dusk conditions. During the experiments, the temperature and relative humidity of the flight tunnel were $23.3^{\circ} \mathrm{C}( \pm 0.72 \mathrm{SD})$ and $23.2 \%( \pm 0.32 \mathrm{SD})$.

All female moths were flown around dusk for the laboratory colony (over a 2-h period, from $1 \mathrm{~h}$ before to $1 \mathrm{~h}$ after dark). We tested individual moths in the flight tunnel, recording behavior for $8 \mathrm{~min}$ per moth. For each moth, we noted whether it left the release cage and made an upwind flight (more than $50 \mathrm{~cm}$ of tight, zigzag flight to within $10 \mathrm{~cm}$ of the target) as well as if it landed on (made contact with) the target. For data analysis, we categorized each moth based on the most complete behavior the moth displayed within an 8-min observation period. Thus, the behavioral responses of moths were categorized as 'no upwind flight' (no directed flight toward the target), 'upwind flight' or 'landing'.

We used freshly cut grape shoots and rubber septa (Thomas Scientific, Swedesboro, NJ, USA) loaded with synthetic blends as targets in the flight tunnel. A grape shoot $(15 \mathrm{~cm})$ was cut and immediately placed into a water pick just prior to flights as described in Cha et al. (2008). Freshly cut shoots were used because we found no difference in upwind flight or landing responses of females to freshly cut shoots or potted grape plants and because we wanted to minimize target size and eliminate possible contamination from pot or soil. In the flight tunnel, the cut shoot was apparent to female GBM. The target for a particular trial was placed at the upwind end in the center of the tunnel (30 $\mathrm{cm}$ from upwind end), affixed on an acetonewashed holder constructed of a glass rod and Teflon tubing. Female moths were introduced in the target plume (determined by using smoke from incense) at about $1.5 \mathrm{~m}$ downwind from the target in the center of the tunnel $(30 \mathrm{~cm}$ from downwind end) in a small metal screen cage.

Field Experiment The field test was conducted during August and September of 2007 in a commercial vineyard near Rushville, NY, USA that had a history of severe GBM damage. We chose a custom-made plastic sheet trap for the field test, based on preliminary flight-tunnel tests of three different trap designs, including the Bio-lure Scenturion delta trap (Suterra LLC, Bend, OR, USA), a red ball trap (Zhang et al. 1999) and our custom plastic sheet trap, and by using the same seven-component blend we used in the field test. The Delta trap and red ball trap baited with the seven-component blend did not catch any upwind flying moths in the flight tunnel, whereas the plastic sheet trap captured $20 \%$ of the moths. The plastic sheet trap was constructed by overlaying 11 layers of plastic sheets (30 $\mathrm{cm} \times 30 \mathrm{~cm}$; Kittrich Co., La Mirada, CA, USA) coated with STP Oil Treatment as the sticky substance. Each week one layer of plastic sheet was removed from each side, allowing us to monitor GBM for up to 6 weeks without applying additional oil. Each trap had six holes $(2.5 \times 2.5 \mathrm{~cm})$ cut in two rows (three holes in top row and three holes in bottom row), with a rubber septum attached at each hole. Each hole was $5 \mathrm{~cm}$ apart and $6.25 \mathrm{~cm}$ from an edge of the trap. Each septum was loaded with $300 \mu$ of one of the synthetic blends or hexane, and attached to the trap with an insect pin. For each hole, a pin was pressed through the middle plastic layer $(2.5 \mathrm{~cm}$ below hole) and woven back out again in a manner that left the point of pin in the center of each hole. Rubber septa were replaced every 3 weeks.

We compared attractiveness of two different lures, the seven- and 11-component blends, as well as a hexane control, to female GBM. Each treatment was replicated four times in four blocks, with two traps installed (one trap per block) at the edge of a vineyard planted with $V$. labrusca (variety niagara) and two traps installed (one trap per block) at the edge of a forest contacting the vineyard. Traps were hung approximately $1 \mathrm{~m}$ from the ground and spaced at least $10 \mathrm{~m}$ apart. Traps were monitored twice weekly from 
August 17, 2007 until September 11, 2007, yielding a total of seven assessments over a 25-day period. Each captured GBM was transferred to the laboratory, and its sex was determined under a dissecting microscope. Since the captured moths were usually drenched with oil, we could not determine whether the female moths were mated or not.

Statistical Analysis The attractiveness of different lure mixtures to female GBM was analyzed by using generalized linear models, with upwind flight or landing as dependent variables and different mixtures as a fixed independent variable, with binomial distribution with logit link function and maximum likelihood estimation (Proc Glimmix; SAS Institute 2006). The effect of the different type of lures (hexane control vs. seven-component vs. 11component) and location of trap (forest edge vs. vineyard edge) on the number of male and female GBM captured over a 25-day period was analyzed by using repeated mixed model analysis of variance with Kenward-Roger degrees of freedom estimation and $\mathrm{AR}(1)$ as a covariance structure for repeated measures (Proc Mixed; SAS Institute 2000). Block was a random factor nested in location. Lure type, location, and time were fixed factors.

\section{Results}

Identification of Candidate Host Volatiles from $V$. riparia Shoots A total of four shoot samples were analyzed by SPME and GC-EAD by using at least five female GBM antennae for each sample. SPME sampling consistently revealed the same 11 significant antennal responses (Table 1). The EAD-active compounds were identified by comparison of mass spectra and GC-MS retention times with those of synthetic standards. The adsorbent collection of volatiles was made from four $V$. riparia shoots and the relative ratios of the $11 \mathrm{EAD}$-active chemicals determined by GC-MS and used in the flight-tunnel tests.

Flight-Tunnel Experiments for Identification of Key Host Volatiles: Negative and Positive Controls Responses of female GBM in the flight tunnel to hexane-loaded rubber septa and grape shoots were used as negative and positive controls, respectively. A total of 42 females were tested individually to a rubber septum loaded with $300 \mu \mathrm{l}$ of hexane; the majority of these did not leave the cage, and those that did flew to the ceiling, sides, or back screen of the flight tunnel. A total of 144 females were flown to grape shoots and $73.6 \%( \pm 3.7 \mathrm{SE})$ of them showed upwind flight. Thus, we used an upwind flight rate of $0 \%$ as our negative control and $73.6 \%$ as our positive control. Some 55.6\% $( \pm 11.7 \mathrm{SE} ; N=51)$ of females tested exhibited upwind flight to the shoot extract; this was not significantly different from the responses to the shoots $\left(t_{24}=1.57 ; P=0.129\right)$.

Flight-Tunnel Experiment 1 Based on the strength of EAD responses (Table 1), we first tested a blend composed of seven compounds, (Z)-3-hexenyl acetate, linalool, $(E)-4,8$ dimethyl-1,3,7-nonatriene, methyl salicylate, $\beta$-caryophyllene, germacrene-D, and $\alpha$-farnesene (treatment 1, Fig. 1). This blend elicited $51.6 \%( \pm 8.9 \mathrm{SE})$ of females to exhibit upwind flight responses, but this was not as attractive as actual grape shoots $\left(t_{36}=2.37 ; P=0.023\right)$. Next, we tested whether there were possible deterrents in this blend by sequentially removing each of the components (treatments 2-8, Fig. 1). No deterrent was detected, as no blend with one component removed had significantly greater attraction

Table 1 EAD-active chemicals from shoots of Vitis riparia determined by SPME, GC-EAD, and GC-MS

\begin{tabular}{|c|c|c|c|c|c|}
\hline Antennae active compounds & GC-MS (\%) & $\begin{array}{l}\text { EAD response } \\
(\mathrm{mV} \pm \mathrm{SD})\end{array}$ & $\begin{array}{l}\text { Relative ratio } \\
\text { in } \operatorname{mix}(\%)\end{array}$ & $\begin{array}{l}\text { First } \\
\text { seven-compo mix }\end{array}$ & $\begin{array}{l}\text { Second } \\
\text { seven-compo mix }\end{array}$ \\
\hline (Z)-3-hexen-1-yl acetate & 14.6 & $0.23 \pm 0.14$ & 10 & $\bullet$ & \\
\hline (Z)-Linalool oxide & 0.7 & $0.14 \pm 0.07$ & 3 & & $\bullet$ \\
\hline (E)-Linalool oxide & 1.2 & $0.09 \pm 0.06$ & 3 & & $\bullet$ \\
\hline Nonanal & 2.2 & $0.06 \pm 0.04$ & 6 & & $\bullet$ \\
\hline Linalool & 1.7 & $0.50 \pm 0.40$ & 6 & $\bullet$ & \\
\hline (E)-4,8-dimethyl 1,3,7-nonatriene & 21.2 & $0.58 \pm 0.42$ & 13 & • & $\bullet$ \\
\hline Methyl salicylate & 1.5 & $0.60 \pm 0.51$ & 6 & - & \\
\hline Decanal & 2.9 & $0.08 \pm 0.08$ & 6 & & $\bullet$ \\
\hline$\beta$-caryophyllene & 10.4 & $0.36 \pm 0.20$ & 3 & $\bullet$ & $\bullet$ \\
\hline Germacrene-D & 10.5 & $0.26 \pm 0.19$ & 11 & $\bullet$ & $\bullet$ \\
\hline$\alpha$-Farnesene & 33.0 & $0.35 \pm 0.21$ & 34 & $\bullet$ & \\
\hline
\end{tabular}

Relative ratio (\%) of peak areas of active compounds in the shoot extract and the strength of antennal response (mV $\pm \mathrm{SD}$ ) to each of the active compounds are shown. All synthetic blends were mixed based on the relative ratio of compounds shown here. In addition, volatile components in the first seven-component blend (see "Flight-tunnel experiment 1") and second seven-component blend (see Flight-tunnel experiment 2) are shown 
Fig. 1 Upwind flight responses (\%) of female grape berry moth $(N=392)$ in flight tunnel to hexane control $(0 \%)$, Vitis riparia shoots (gray bar), and various synthetic blends (white bars). Treatment 1 was a mixture of seven compounds listed below the graph. Treatments $2-9$ were subsets of treatment 1 . At least 20 individual moths were flown to each synthetic blend tested. Different letters on bars indicate significant differences $(P<0.05$; *tDMNT: $(E)-4,8$-dimethyl-1,3,7-nonatriene)

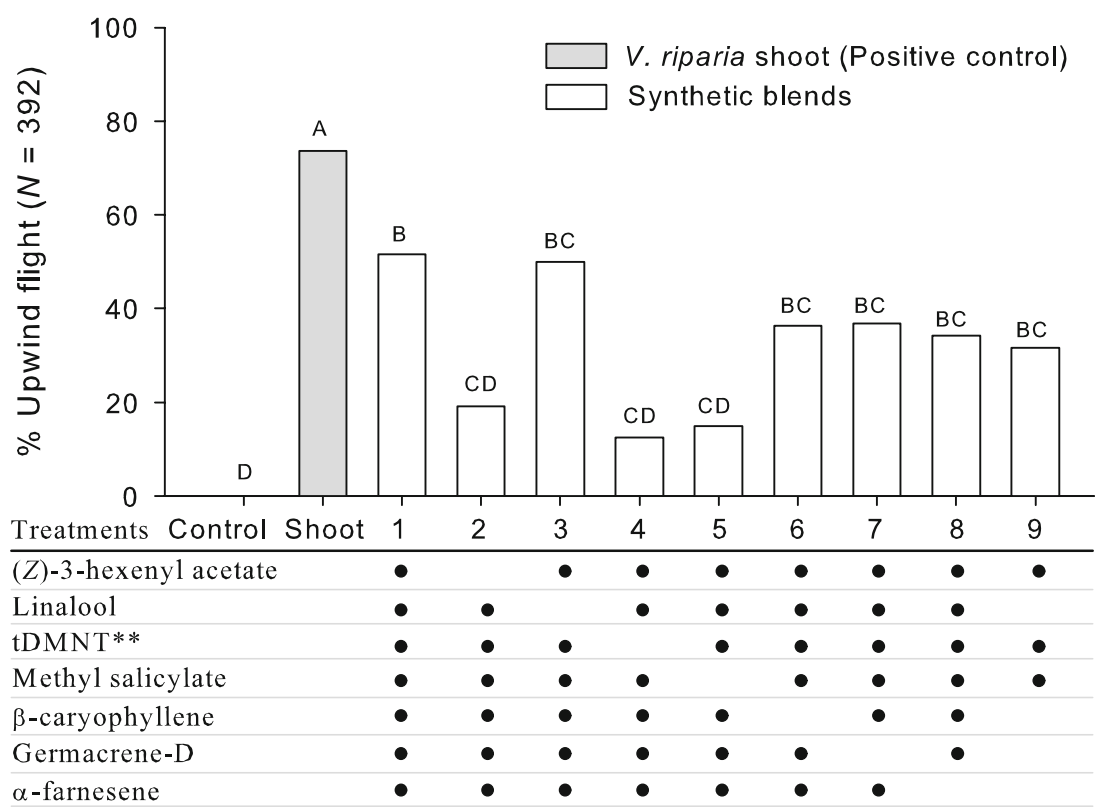

compared to the original seven-component blend. However, from this experiment, (Z)-3-hexenyl acetate, (E)-4,8-dimethyl-1,3,7-nonatriene, and methyl salicylate appeared to be important components, since removing any of these resulted in significantly fewer upwind flights compared to the original blend (Fig. 1). The blend composed only of ( $Z$ )3-hexenyl acetate, (E)-4,8-dimethyl-1,3,7-nonatriene, and methyl salicylate (treatment 9) elicited GBM flight responses not statistically different from those to the original blend $\left(t_{36}=1.37 ; P=0.179\right)$. Three hundred and ninety two females were tested in this experiment.

Flight-Tunnel Experiment 2 The second set of flight-tunnel trials used all 11 EAD-active compounds listed in Table 1. This full blend elicited $68.6 \%( \pm 7.8 \mathrm{SE})$ of females to exhibit upwind flight; this was not statistically different from that to grape shoots $\left(t_{38}=0.60 ; P=0.55\right.$; treatment 10 , Fig. 2). The removal from the blend of linalool, $\beta$ -
Fig. 2 Upwind flight responses (\%) of female grape berry moth $(N=431)$ in flight tunnel to Vitis riparia shoots (gray bar) and various synthetic blends (white bars). Treatment 10 was a mixture of 11 compounds listed below the graph. Treatments 11-19 were various subsets of treatment 10. At least 20 individual moths were flown to each synthetic blend tested. Different letters on bars indicate significant differences $(P<0.05)$, except that $P=0.051$ for both "shoot vs. treatment 11 " and "treatment 17 vs. treatment 18 " (*tDMNT: $(E)$-4,8-dimethyl1,3,7-nonatriene)

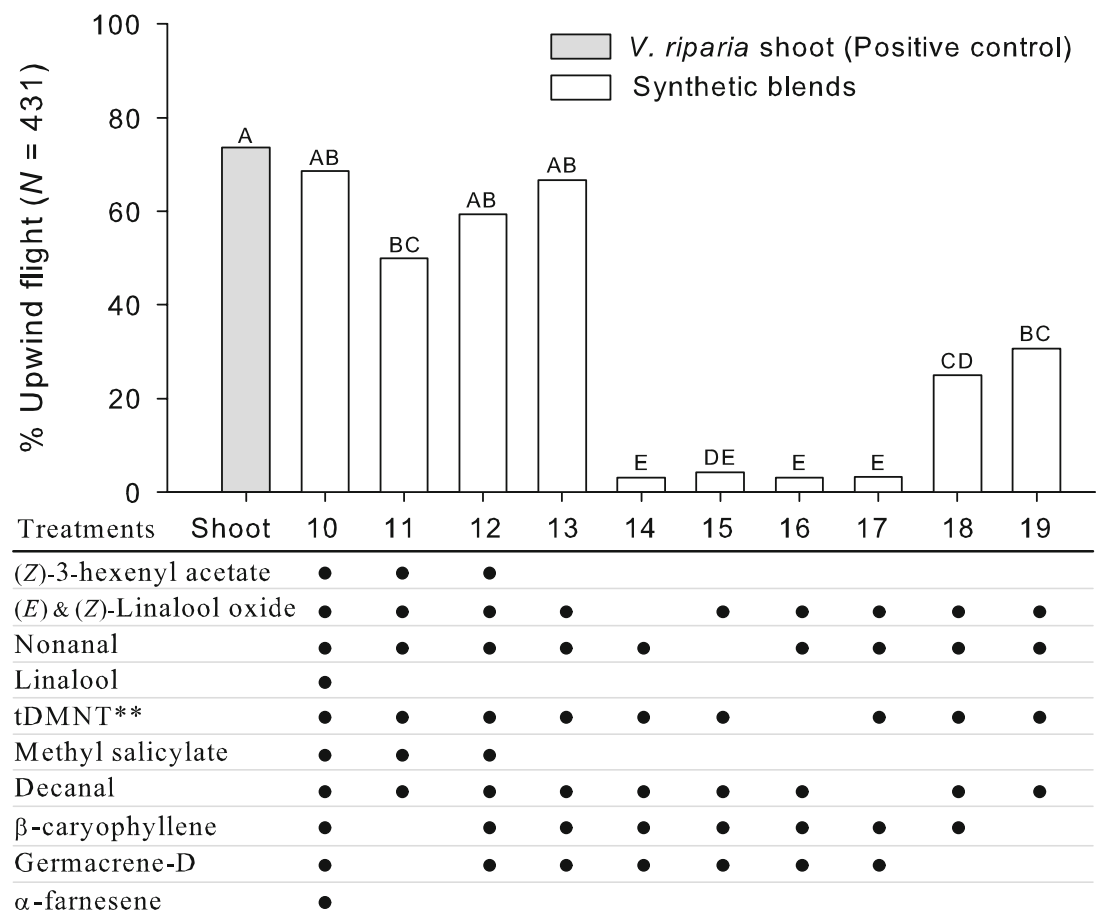




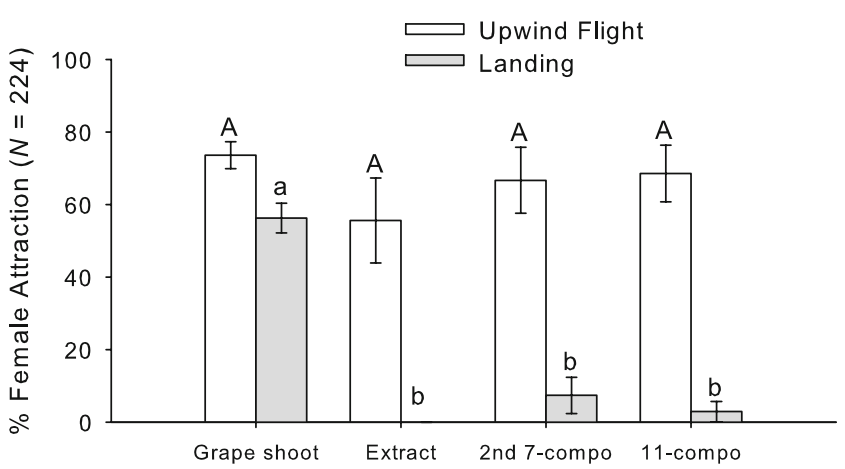

Fig. 3 Comparison of upwind flight (\%) and landing (\%) responses of female grape berry moth to Vitis riparia shoots, shoot extract and two synthetic blends. 11-component blend $=(Z)-3$-hexen-1-yl acetate, $(E)$-linalool oxide, (Z)-linalool oxide, nonanal, linalool, $(E)-4,8$ dimethyl-1,3,7-nonatriene, methyl salicylate, decanal, $\beta$-caryophyllene, germacrene-D, and $\alpha$-farnesene; second seven-component blend=11-component blend minus (Z)-3-hexen-1-yl acetate, linalool, methyl salicylate and $\alpha$-farnesene. Different letters (capital letters for orientation response and small letters for landing response) on bars indicate significant differences $(P<0.05)$. Error bars $= \pm 1 \mathrm{SE}$

caryophyllene, germacrene-D, and $\alpha$-farnesene, the four compounds that appeared not to be important based on the results of the first experiment, resulted in $50 \%( \pm 11.8 \mathrm{SE})$ of GBM tested exhibiting upwind flight; this was not statistically different from the responses exhibited to the 11component blend $\left(t_{38}=1.31 ; P=0.198\right)$, but was marginally lower than that of the shoots $\left(t_{38}=2.02 ; P=0.051\right)$. Adding $\beta$-caryophyllene and germacrene-D back to the blend (treatment 12) increased attractiveness to the level comparable to shoots.

In a further treatment, (Z)-3-hexen-1-yl acetate and methyl salicylate (treatment 13) were removed from the nine-component blend (i.e., 11-component blend minus linalool and $\alpha$-farnesene) without reducing the attractiveness $(66.7 \% \pm 9.1 \mathrm{SE})$ of the mixture relative to that of the shoots or the original 11-component blend (Fig. 2). Linalool oxide, nonanal, (E)-4,8-dimethyl-1,3,7-nonatriene, and decanal were key components in this second sevencomponent blend. The removal of linalool oxide (as a 1:1 mixture of $(E)$ - and $(Z)$-linalool oxide), nonanal, decanal, or (E)-4,8-dimethyl-1,3,7-nonatriene individually resulted in significantly lower (3.1 4.2\%; treatments $14-17$, Fig. 2) flight responses from females. In contrast, the removal of germacrene-D (treatment 18) or $\beta$-caryophyllene and germacrene-D (treatment 19) from the second nine-component blend resulted in only a $41.7 \%$ and $36.1 \%$ decrease (relative to that to the second seven-component blend), respectively, in upwind flight responses of females. Since the germacrene-D contained $9 \%$ of $\beta$-caryophyllene, however, we could not conclude that both germacrene-D and $\beta$-caryophyllene were essential compounds in the second seven-component blend. Four hundred and thirty one females were tested in this experiment.
Although the upwind flight responses of females to the 11and second seven-component synthetic blends (treatments 10 and 13 in Fig. 2, respectively) were not significantly different $\left(F_{3,24}=0.93 ; P=0.442\right)$ from those to grape shoots or shoot extract, there were significant differences among these treatments in terms of landing $\left(F_{3,24}=8.71 ; P<0.001\right)$. In particular, the extract and synthetic blends elicited significantly lower landings from females in the flight tunnel compared to the shoots (Fig. 3). Two hundred and twenty four females were tested in this experiment.

Field Experiment In a late-season field trial, conducted in a high GBM-pressure commercial vineyard, traps baited with either the 11- or second seven-component blends caught significantly $\left(F_{2,15}=7.22 ; P=0.006\right)$ higher numbers of female GBM than traps baited with a hexane control (Fig. 4); there was no significant effect of location (i.e., forest edge vs. vineyard) or time on catch of female moths. In contrast, there were no differences among treatments in the numbers of male moths captured $\left(F_{2,24.9}=0.08 ; P=\right.$ 0.925). For males, however, we found a significant interaction among time, location, and lure $\left(F_{12,27}=2.47\right.$; $P=0.025)$. Overall, the total number of female and male moths captured was low ( 0.25 moths per day for best treatment), especially considering that the trial was performed in a high GBM-pressure vineyard. The best blend caught an average of four female moths/trap during the 25-day trapping period.

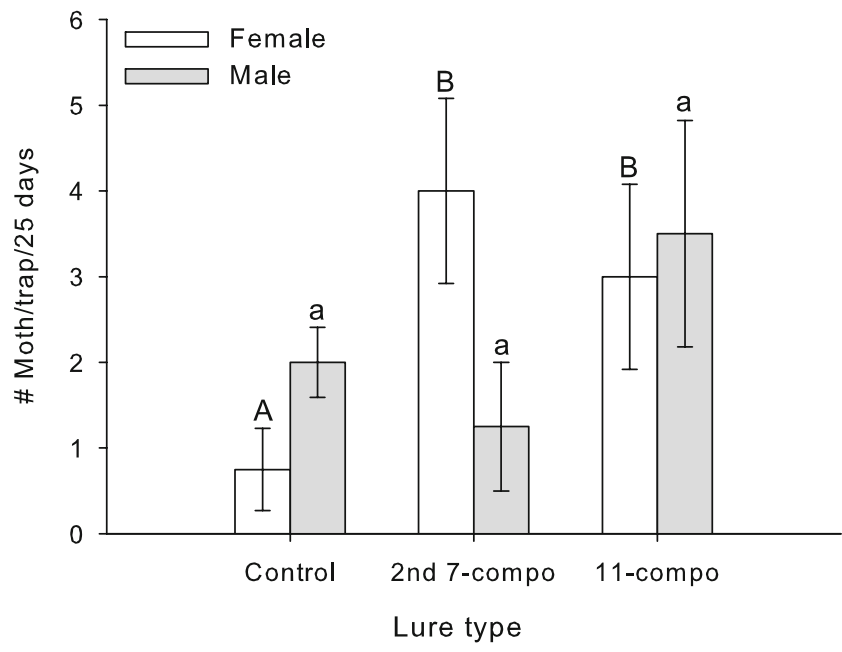

Fig. 4 Number of female and male grape berry moth captured in plastic-sheet traps, each baited with six rubber septa impregnated with a hexane control, the second seven-component blend or an 11component blend in a commercial vineyard over a 25-day period. Eleven-component blend= $(Z)$-3-hexen-1-yl acetate, $(E)$-linalool oxide, $(Z)$-linalool oxide, nonanal, linalool, $(E)$-4,8-dimethyl-1,3,7-nonatriene, methyl salicylate, decanal, $\beta$-caryophyllene, germacrene- $D$, and $\alpha$-farnesene; second seven-component blend=11-component blend minus (Z)-3-hexen-1-yl acetate, linalool, methyl salicylate and $\alpha$-farnesene. Error bars $= \pm 1 \mathrm{SE}$ 


\section{Discussion}

We identified a blend of 11 volatile compounds [(Z)-3hexen-1-yl acetate, $(E)$-linalool oxide, $(Z)$-linalool oxide, nonanal, linalool, $(E)$-4,8-dimethyl-1,3,7-nonatriene, methyl salicylate, decanal, $\beta$-caryophyllene, germacrene- $D$, and $\alpha$-farnesene] from the shoots of $V$. riparia, a native host, that elicited an equivalent percentage of upwind flight by female GBM as that by intact shoots. Traps baited with this blend successfully caught female GBM in a vineyard. Subtraction of (Z)-3-hexen-1-yl acetate, linalool, methyl salicylate, and $\alpha$-farnesene from this blend did not affect efficacy of the mixture with respect to eliciting both upwind flight responses of female GBM in a flight tunnel and trap catch in the field.

We originally hypothesized that the magnitude of antennal response in the GC-EAD analysis may indicate the relative importance (i.e., behavioral activity) of each of the compounds. However, this approach resulted in a blend that was not as attractive as grape shoots in our first set of flight-tunnel experiments. Addition of the four compounds that elicited the smallest antennal responses resulted in improved levels of upwind flight, comparable to that to grape shoots in the second set of experiments. This result suggests that the strength of antennal responses should not be assumed to correspond directly to behavioral activity.

Although both the 11- and second seven-component blends elicited upwind flight responses of female GBM comparable to that to grape shoots, the lower level of landing and low trap catches in the field to the synthetic blends suggest that their compositions may not be optimal for trapping female GBM. Several, non-mutually exclusive factors may be responsible for this. GBM could use both olfactory and visual cues for landing. For example, in Lygus hesperus, visual cues were shown to enhance the response to host-plant volatiles (Blackmer and Cañas 2005). GBM, a crepuscular species, oviposits dramatically less in the absence of light (Clark and Dennehy 1988), suggesting that females may use visual cues in this behavior. In addition, other chemicals, such as fatty acids, with low volatility present on grape leaf or berry surfaces could also elicit landing. For example, Wallace et al. (2004) reported that mated females of the spruce budworm preferentially oviposited on substrates treated with waxes from the needles of a host plant. Finally, $\mathrm{CO}_{2}$ and moisture gradients released by grape shoots could affect close-range landing behaviors (references in Thom et al. 2004; Hilker and McNeil 2008).

The low-landing responses we obtained to the synthetic mixtures could also be due to a sub-optimal ratio of components in the blend. It has been suggested that many herbivores and parasitoids perceive a specific mixture of plant volatiles to locate their host reliably (Schoonhoven et al. 1998; Hilker and McNeil 2008). Such specificity can be mediated by species-specific host chemicals. However, the compounds in our two blends are relatively ubiquitous plant volatiles. In such a case, a species-specific ratio of common compounds could be a key to locating hosts within a complex environment of plant volatiles (Bruce et al. 2005; Hilker and McNeil 2008). Although we based the ratio of compounds in the blends on our adsorbent collections from live, potted plants, it is possible that the ratio of components we tested might not correspond exactly to that released by live grape shoots due to the adsorbent characteristics (Tasin et al. 2006b), and also the differential release rate of components from the rubber septa (Butler and McDonough 1979; Heath et al. 1986). The fact that we also observed low levels of landings on septa with the extract supports this contention, although other factors may also be involved.

Headspace volatiles were collected from live potted grapes to ensure that they were consistent in quality and quantity with that released by plants in the field, since physical wounding, such as cutting, could cause changes in volatile emissions both locally and systemically (Davies 1987; Rhodes et al. 1999). In the flight tunnel, however, we found both cut shoots and potted plants were equally attractive to female GBM, suggesting that cut-plant materials did not significantly affect behavior. However, immediately after excision, there was a six-fold increase in (Z)-3-hexenyl acetate released by shoots. This chemical, a green leaf volatile, is well recognized as a wound-induced compound (e.g., Engelberth et al. 2004), and also elicited a GC-EAD response from female GBM. Thus, the ratio of at least one GC-EAD-active compound, (Z)-3-hexenyl acetate, that was also considered essential in one of our blends (see below), appears to be not critical for female GBM behavior. Our future research will investigate explicitly the role of blend ratio of GC-EAD-active compounds to determine how GBM, a specialist herbivore, recognizes its host by using common, ubiquitous volatile compounds, and, if ratio is important, whether the ratios of all the GC-EAD compounds are important or just that of a subset of the compounds.

The results from the field-trapping experiment showed no significant differences in the number of males captured between traps with synthetic lures based on host-plant volatiles and control traps. This suggests that males may not be responding to the same blend of host-plant volatiles as females, similar to the findings in flight-tunnel trials for the European berry moth (e.g., Masante-Roca et al. 2007). However, definitive conclusions cannot be drawn since our field trial was conducted late in the season, and relatively few moths were captured.

Not all the volatile compounds isolated by GC-EAD were essential for eliciting upwind flight from female 
GBM. Moreover, what compounds are essential appears to be context specific (e.g., Mumm and Hilker 2005; Hilker and McNeil 2008). For example, removal of (Z)-3-hexen-1yl acetate, linalool, methyl salicylate, and $\alpha$-farnesene from the 11-component blend gave equivalent levels of attraction as shoots. Removal of any of the remaining seven compounds, individually, significantly reduced attractiveness of the blend, suggesting that some or all of these seven compounds $[(E)$-linalool oxide, $(Z)$-linalool oxide, nonanal, (E)-4,8-dimethyl-1,3,7-nonatriene, decanal, $\beta$-caryophyllene, and germacrene-D] are essential for optimal attraction. However, with the first seven-component blend of (Z)-3hexen-1-yl acetate, linalool, (E)-4,8-dimethyl-1,3,7-nonatriene, methyl salicylate, $\beta$-caryophyllene, germacrene-D and $\alpha$-farnesene that we tested, we found that $(Z)-3$-hexen1-yl acetate, $(E)$-4,8-dimethyl-1,3,7-nonatriene and methyl salicylate were essential components of the blend. Therefore, the finding that some compounds, such as (Z)-3hexen-1-yl acetate and methyl salicylate in this study, acted as essential components in one blend (i.e., the first sevencomponent blend; treatment 1 in Fig. 1) but not in another (i.e., the second seven-component blend; treatment 13 in Fig. 2) suggests that some plasticity exists in the volatilebased host recognition system of GBM. Such a context dependency may have important implications both for understanding the evolution of volatile-based host recognition of herbivores and for developing a synthetic lure that works in the field. Plasticity in recognition of host plants with different volatile blends could have been selected for to allow host location under seasonal and daily variation in host chemistry (e.g., Vallat and Dorn 2005; Hilker and McNeil 2008).

The long-term goal of our research is to develop a synthetic lure based on host-plant volatiles that can be used to monitor female GBM populations in the field as a way to improve the timing of management decisions. Our lateseason field trial provides some reason for optimism that this approach is feasible. However, the low capture of moths indicates that significant hurdles remain. One problem is that synthetic lures may not stand out to females from the background volatile noise produced by the crop plant and the many other plants in the environment. Ultimately, we may need to discover some novel compounds that are uniquely attractive, either temporally or spatially. Furthermore, information on close-range visual and chemosensory cues may help improve the utility of volatile chemical-based lures for pest management.

Acknowledgments We thank Sara Villani, Charles Moser, Eric Smith, Shinyoung Park, Rachel Tucker, Mike Colizzi, Jessica Worden, Arianna Waheed, and Kevin Conley, for support on various aspects of this research, but particularly their efforts in maintaining the GBM colony and setting up mating cohorts. The manuscript was improved by comments from two anonymous reviewers and the subject editor for which we are grateful. We thank Aijun Zhang and Peter Teal for the gift of the $(E)$-4,8-dimethyl-1,3,7-nonatriene and germacrene-D, respectively. Authors also thank Arthur Agnello for plastic sheet trap design. Paul Robbins and Shannon Olsson provided valuable technical insights in the beginning of this study. Special thanks go to David Wise at Cornell University Glass Workshop for constructing volatile collection chamber and Jeff and June Pendleton for permitting us to perform field trial in their commercial vineyard. This research was supported by USDA NRI grant \#2005-35302-16154 and USDA Viticultural Consortium.

\section{References}

Angioy, A. M., Desogus, A., Barbarossa, I. T., Anderson, P., and HANSSON, B. S. 2003. Extreme sensitivity in an olfactory system. Chem. Sens. 28:279-284.

Ansebo, L., Coracini, M. D. A., Bengtsson, M., Liblikas, I., Ramirez, M., Borg-Karlson, A. K., TAsin, M., and WitzGall, P. 2004. Antennal and behavioural response of codling moth Cydia pomonella to plant volatiles. J. Appl. Entomol. 128:488493.

Bernays, E. A., and Chamman, R. F. 1994. Host plant selection by phytophagous insects. Chapman \& Hall, New York.

BlaCKMER, J. L., and CAÑAS, L. A. 2005. Visual cues enhance the response of Lygus hesperus (Heteroptera: Miridae) to volatiles from host plants. Environ. Entomol. 34:1524-1533.

Blackmer, J. L., Rodriguez-SaOnA, C., Byers, J. A., Shope, K. L., and SMITH, J. P. 2004. Behavioral response of Lygus hesperus to conspecifics and headspace volatiles of alfalfa in a Y-tube olfactometer. J. Chem. Ecol. 30:1547-1564.

Bruce, T. J. A., Wadhams, L. J., and Woodcock, C. M. 2005. Insect host location: a volatile situation. Trend. Plant Sci. 10:269-274.

ButLER, L. I., and MCDONOUGH, L. M. 1979. Insect sex-pheromonesevaporation rates of acetates from natural-rubber septa. J. Chem. Ecol. 5:825-837.

Cha, D. H., Hesler, S. P., Moser, C. L., Nojima, S., Linn, C. E., RoElofs, W. L., and LoEB, G. M. 2008. Flight tunnel responses of female grape berry moth (Paralobesia viteana) to host plants. J. Chem. Ecol. 34:622-627.

Clark, L. G., and DenNehy, T. J. 1988. Oviposition behavior of grape berry moth. Entomol. Exp. Appl. 47:223-230.

Coracini, M., Bengtsson, M., Liblikas, I., and WitzGall, P. 2004. Attraction of codling moth males to apple volatiles. Entomol. Exp. Appl. 110:1-10.

DAVIES, E. 1987. Wound responses in plants, pp, pp. 243-264, in D. Davies (ed.). The Biochemistry of PlantsAcademic, London.

DozIER, H. L., and ButLer, H. G. 1929. Life history and control of the grape berry moth in Delaware. J. Econom. Entomol. 22:132136.

Engelberth, J., Alborn, H. T., Schmelz, E. A., and Tumlinson, J. H. 2004. Airborne signals prime plants against insect herbivore attack. PNAS USA 101:1781-1785.

EPHrussi, B., and BEADLE, G. W. 1936. A technique for transplantation of Drosophila. Am. Nat. 70:218-225.

GLEISSNER, B. D. 1943. Biology and control of berry moth in the Erie grape belt. Pennsylvania State College School of Agriculture Bulletin 451:1-74.

GoodwIN, W. H. 1916. The grape berry moth. Ohio Agricultural Experiment Station Bulletin 293:259-307.

Greenwald, R., Chaykovsky, M., and Corey, E. J. 1963. TheWittig reaction using methylsulfinyl carbanion-dimethyl sulfoxide. $J$. Org. Chem. 28:1128-1129. 
HAMMACK, L. 2003. Volatile semiochemical impact on trapping and distribution in maize of northern and western corn rootworm beetles (Coleoptera: Chrysomelidae). Agri. For. Entomol. 5:113122.

Heath, R. R., Teal, P. E. A., Tumlinson, J. H., and Mengelkoch, L. J. 1986. Prediction of release ratios of multicomponent pheromones from rubber septa. J. Chem. Ecol. 12:2133-2143.

Hern, A., and DORN, S. 2004. A female-specific attractant for the codling moth, Cydia pomonella, from apple fruit volatiles. Naturwissenschaften 91:77-80.

HILKER, M., and MCNEIL, J. 2008. Chemical and behavioral ecology in insect parasitoids: how to behave optimally in a complex odourous environment, pp. 92-112, in E. Wajnberg, C. Bernstein, and J. van Alphen (eds.). Behavioral Ecology of Insect ParasitoidsBlackwell, Malden, MA.

Hoffman, C. J. 1990. Development and validation of a risk assessment program for the management of grape berry moth, Endopiza viteana (Clemens), in New York state. PhD Dissertation, Cornell University, Ithaca, NY.

Leskey, T. C., ZhANG, A. J., and HerZOG, M. 2005. Nonfruiting host tree volatile blends: novel attractants for the Plum curculio (Coleoptera: Curculionidae). Environ. Entomol. 34:785-793.

Masante-Roca, I., Anton, S., Delbac, L., Dufour, M.-C., and GADENNE, C. 2007. Attraction of the grapevine moth to host and non-host plant parts in the wind tunnel: effects of plant phenology, sex, and mating status. Entomol. Exp. Appl. 122:239-245.

Mitchell, V. J., Manning, L.- A. , Cole, L., Suckling, D. M., and EL-SAYED, A. M. 2008. Efficacy of the pear ester as a monitoring tool for codling moth Cydia pomonella (Lepidoptera: Tortricidae) in New Zealand apple orchards. Pest Manage. Sci. 64:209-214.

MumM, R., and HiLKER, M. 2005. The significance of background odour for an egg parasitoid to detect plants with host eggs. Chem. Sens. 30:337-343.

Nagarkatti, S., MuZa, A., and Saunders, M. 2000. Meridic diet for Endopiza viteana (Lepidoptera: Tortricidae). Can. Entomol. 132:259-261.

Natale, D., Mattiacci, L., Pasqualini, E., and Dorn, S. 2004. Apple and peach fruit volatiles and the apple constituent butyl hexanoate attract female oriental fruit moth, Cydia molesta, in the laboratory. J. Appl. Entomol. 128:22-27.

Nojima, S., Linn, C., Morris, B., Zhang, A. J., and Roelofs, W. 2003. Identification of host fruit volatiles from hawthorn (Crataegus Spp.) attractive to hawthorn-origin Rhagoletis pomonella flies. J. Chem. Ecol. 29:321-336.

Pinero, J. C., Jacome, I., VARgas, R., and Prokopy, R. J. 2006. Response of female melon fly, Bactrocera cucurbitae, to hostassociated visual and olfactory stimuli. Entomol. Exp. Appl. 121:261-269.

Rhodes, J. D., Thain, J. F., and Wildon, D. C. 1999. Evidence for physically distinct systemic signalling pathways in the wounded tomato plant. Ann. Bot. 84:109-116.

Roelofs, W. L., Tette, J. P., Taschenberg, E. F., and Comeau, A. 1971. Sex pheromone of the grape berry moth: identification by classical and electroantennogram methods, and field tests. $J$. Insect Physiol. 17:2235-2243.

SAS Institute. 2000. SAS/STAT User's Guide. SAS Institute, Cary, North Carolina, USA.

SAS Institute. 2006. The GLIMMIX Procedure. <http://www.sas. com>.

SCHOONHOVEN, L. M., JeRmy, T., and van LOON, J. J. A. 1998 Insect-Plant Biology. Chapman \& Hall, London, UK.

SLingerland, M. V. 1904. The grape berry moth. Cornell University, Agricultural experiment station of the college of agriculture Bulletin 223:43-59.

TASCHENBERG, E. F. 1945. The biology and control of the grape berry moth Polychrosis vineana (Clemens). PhD Dissertation, Cornell University, Ithaca, NY.

Tasin, M., Anfora, G., Ioriatti, C., Carlin, S., De Cristofaro, A., Schmidt, S., Bengtsson, M., Versini, G., and WitzGall, P. 2005. Antennal and behavioral responses of grapevine moth Lobesia botrana females to volatiles from grapevine. J. Chem. Ecol. 31:77-87.

Tasin, M., Backman, A. C., Bengtsson, M., Ioriatti, C., and WitzGALl, P. 2006a. Essential host plant cues in the grapevine moth. Naturwissenschaften 93:141-144.

Tasin, M., Backman, A. C., Bengtsson, M., Varela, N., Ioriatti, C., and WitZGALL, P. 2006b. Wind tunnel attraction of grapevine moth females, Lobesia botrana, to natural and artificial grape odour. Chemoecology 16:87-92.

Tasin, M., Backman, A.-C., Coracini, M., CASAdo, D., Ioriatti, C., and WitzGaLl, P. 2007. Synergism and redundancy in a plant volatile blend attracting grapevine moth females. Phytochemistry 68:203-209.

Thom, C., Guerenstein, P. G., Mechaber, W. L., and Hildebrand, J. G. 2004. Floral $\mathrm{CO}_{2}$ reveals flower profitability to moths. $J$. Chem. Ecol. 30:1285-1288.

VAllat, A., and DORN, S. 2005. Changes in volatile emissions from apple trees and associated response of adult female codling moths over the fruit-growing season. J. Ag. Food Chem. 53:4083-4090.

VISSER, J. H. 1986. Host odor perception in phytophagous insects. Annu. Rev. Entomol. 31:121-144.

Wallace, E. K., Albert, P. J., and Mcneil, J. N. 2004. Oviposition behavior of the eastern spruce budworm Choristoneura fumiferana (Clemens) (Lepidoptera: Tortricidae). J. Insect Behavior 17:145154.

Weigle, T., BixBy, J., and English-LoeB, G. 1999. Reexamination of grape berry moth management practices in the Lake Erie region. 1998 New York State Fruit Project Reports Relating to IPM. NYS IPM Publication \#216. Cornell University Cooperative Extension.

Zhang, A. J., Linn, C., Wright, S., Prokopy, R., Reissig, W., and ROELOFS, W. 1999. Identification of a new blend of apple volatiles attractive to the apple maggot, Rhagoletis pomonella. $J$. Chem. Ecol. 25:1221-1232.

Zhang, A., Oliver, J. E., Aldrich, J. R., WANG, B., and Mastro, V. C. 2002. Stimulatory beetle volatiles for the Asian longhorned beetle, Anoplophora glabripennis (Motschulsky). Z. Naturforsch. 57c:553-558. 